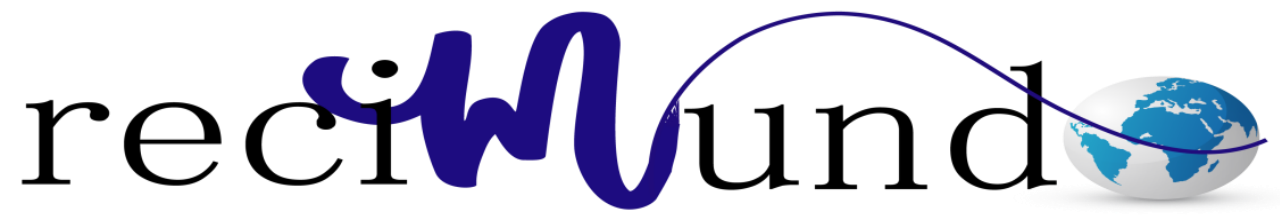

Revista Científica Mundo de la Investigación y el Conocimiento

Sofía Lissette Pacherres Nolivos ${ }^{\text {a }}$; Eugenia Estefanía Alvarado Palma ${ }^{\text {b; }}$ Irma Elizabeth Naranjo Peña ${ }^{\text {c}}$; David Benavides López ${ }^{\mathrm{d}}$

La Universidad Ecuatoriana ante las nuevas Profesiones

Revista Científica Mundo de la Investigación y el Conocimiento. Vol. 2 núm.2, mayo, ISSN: 2588-073X, 2018, pp. 742-760

DOI: 10.26820/recimundo/2.(2).2018.742-760

Editorial Saberes del Conocimiento

Recibido: 05/12/2017

Aceptado: 15/02/2018

a. Universidad de Guayaquil;

b. Licenciada en Ciencias de la Educación Mención Informática; Cursando Maestría Gestión Educativa; Ministerio de Educación Unidad Educativa Assad Bucaram Elmhalin; 1ulu12360@ hotmail.com

c. Universidad de Guayaquil; Irma_naranjo1218@hotmail.com

d. Universidad de Guayaquil; dbenavides6@hotmail.com 


\section{La Universidad Ecuatoriana ante las nuevas Profesiones}

Vol. 2, núm. 2., (2018)

Sofía Lissette Pacherres Nolivos; Eugenia Estefanía Alvarado Palma; Irma Elizabeth Naranjo Peña; David Benavides López

\section{RESUMEN}

Se sabe que la educación de los ciudadanos de un país, en todos sus niveles, es parte fundamental para el desarrollo sociocultural, económico y sostenible del mismo. La experiencia en las naciones que hoy en día alcanzan mayores avances en cuanto a ciencia, tecnología, salud, calidad de vida, estabilidad económica, entre otros aspectos, comparten la característica de poseer sistemas educativos de avanzada, por estar progresivamente adaptados a las necesidades tanto de sus ciudadanos como del mundo globalizado. En base a esta realidad, se estimó desarrollar la presente investigación, que tuvo como objetivo principal, describir los aspectos más resaltantes del Sistema de Educación Superior en Ecuador que existe tras el Nuevo Marco Constitucional de 2008 y la promulgación de la Ley Orgánica de Educación Superior (LOES), y en segunda instancia, emitir una opinión respecto a si el mencionado sistema se adapta a las posibles demandas de carreras para nuevas profesiones que emergen en el marco de la globalización y los avances tecnológicos. La naturaleza de esta investigación es documental, de tipo descriptiva, a nivel exploratorio, por cuanto la misma se ha limitado a la revisión e interpretación de documentos, a los que se tuvo acceso tanto de manera física como digital, en éstos últimos, a través del motor de búsqueda de Google Académico® y Google ${ }^{\circledR}$, siendo con dicha bibliografía que se pudo construir, a manera de conclusión, un criterio propio que responde a la interrogante aquí planteada de manera medianamente favorable, ya que para obtener resultados verdaderamente concluyentes, tendría que desarrollarse otra investigación más profunda con la cual justificar tal pretensión, de tal manera que, queda de parte del lector valorar la información aquí ofrecida para así aceptar o rechazar el contenido del mismo.

Palabras clave: Educación, desarrollo, necesidades, profesiones, avances. 


\section{La Universidad Ecuatoriana ante las nuevas Profesiones}

Vol. 2, núm. 2., (2018)

Sofía Lissette Pacherres Nolivos; Eugenia Estefanía Alvarado Palma; Irma Elizabeth Naranjo Peña; David Benavides López

\section{ABSTRACT}

It is known that the education of the citizens of a country, in all its levels, is a fundamental part for the sociocultural, economic and sustainable development of it. The experience in the nations that today achieve greater advances in terms of science, technology, health, quality of life, economic stability, among other aspects, share the characteristic of having advanced educational systems, because they are progressively adapted to the needs of both of its citizens as of the globalized world. Based on this reality, it was estimated to develop the present investigation, which had as main objective, to describe the most outstanding aspects of the Higher Education System in Ecuador that exists after the New Constitutional Framework of 2008 and the promulgation of the Organic Law of Higher Education (LOES), and secondly, to issue an opinion on whether the aforementioned system is adapted to the possible demands of careers for new professions that emerge within the framework of globalization and technological advances. The nature of this research is documentary, descriptive, exploratory level, as it has been limited to the review and interpretation of documents, which were accessed both physically and digitally, in the latter, through of the search engine of Google Academic $₫$ and Google ${ }^{\circledR}$, being with this bibliography that it was possible to construct, by way of conclusion, an own criterion that responds to the question posed here in a moderately favorable way, since to obtain truly conclusive results, it would have than to develop another deeper investigation with which to justify such a claim, in such a way that, it is up to the reader to value the information offered here in order to accept or reject the content of it.

Keywords: Education, development, needs, professions, advances. 


\section{La Universidad Ecuatoriana ante las nuevas Profesiones}

Vol. 2, núm. 2., (2018)

Sofía Lissette Pacherres Nolivos; Eugenia Estefanía Alvarado Palma; Irma Elizabeth Naranjo Peña; David Benavides López

\section{Introducción.}

La transformación de las universidades se produce en respuesta a la influencia que ejercen, tres factores preponderantes, como lo son: la globalización, el desarrollo tecnológico y la competitividad. Esto es de cara a los constantes cambios que se producen cada día alrededor del contexto tecnológico en el que se desenvuelve la sociedad a nivel mundial, por esta razón es importantísimo que los entes responsables adelanten como estrategia de gestión: la planeación, que apoyada en esquemas estratégicos generan dinámicas más constructivas y efectivas en sus funciones sustantivas, administrativas y financieras.

Autores como Cobos (2018) afirman que el cambio en el progreso de los países y en el aumento de la calidad de vida de sus pueblos, se encuentra ampliamente vinculado a la educación universitaria. Agrega que es uno de los medios culturales que permite convivir en un constante proceso de interacción con el entorno, descubriendo y cultivando su individualidad como país y fortaleciendo su convivencia en colectividad.

En los últimos años el Estado ecuatoriano ha impulsado fuertemente y orientado sus esfuerzos por lograr una transformación real de sus universidades, sobre la base de una educación superior de calidad con niveles de excelencia. (EcuadorUniversitario.com, 2016)

En la obra de Macías \& Mendoza (2016), respecto al sistema de Educación Superior en el Ecuador afirman que:

En efecto, el sistema está aprendiendo a desaprender la cultura estática de falta de calidad en la que estaba inmersa y ha emprendido un movimiento de cambio que compromete al 


\section{La Universidad Ecuatoriana ante las nuevas Profesiones}

Vol. 2, núm. 2., (2018)

Sofía Lissette Pacherres Nolivos; Eugenia Estefanía Alvarado Palma; Irma Elizabeth Naranjo Peña; David Benavides López

Estado y a la sociedad a asumir nuevos retos para apoyar dichos cambios que aportarán, sin duda, al desarrollo humano integral y, por ende, a una mejor calidad de vida de sus miembros así como al progreso de la sociedad y del país, reflexión generada desde el marco legal y la evolución de la Universidad en función de indicadores cualicuantitativos que permiten visualizar el compromiso de esta con la evolución del mundo. (p.90)

A partir de la aprobación en el año 2010 de la Ley Orgánica de Educación Superior, la universidad ecuatoriana ha sido expuesta a nuevos retos y desafíos que comprometen a cada uno de los tejidos institucionales participantes en su gestión.

De acuerdo a lo publicado por (EcuadorUniversitario.com, 2016) se conoce que desde hace aproximadamente dos años, en Ecuador se lleva a cabo el Proyecto de Reconversión de la Educación Superior Técnica y Tecnológica, y en el marco de éste, se empezó a implementar Institutos Técnicos y Tecnológicos Superiores Sectoriales (INTES), que contarán con oferta especializada hacia un sector priorizado de la producción, industrias estratégicas y de interés público en áreas determinadas del conocimiento, tales como: Desarrollo de Software; Guiatura Turística; Atención Primaria de Salud; Seguridad Ciudadana y Orden Público; Minería subterránea; Logística en Almacenamiento y Distribución; Mecatrónica Automotriz; Plásticos; Química; Confección Textil, entre otras.

Esto, aunque está concebido más que todo para cubrir la creciente demanda flotante del sistema principal, también podría entenderse como un primer vestigio de mayor apertura para el surgimiento de nuevas profesiones en el sistema de Educación Superior. 


\section{La Universidad Ecuatoriana ante las nuevas Profesiones}

Vol. 2, núm. 2., (2018)

Sofía Lissette Pacherres Nolivos; Eugenia Estefanía Alvarado Palma; Irma Elizabeth Naranjo Peña; David Benavides López

\section{Materiales y métodos.}

Para esta investigación se destinó el uso de un computador personal con acceso a internet como recurso fundamental, por medio del cual se pudo acceder a distintas fuentes de información digital ubicada en bases de datos nacionales y extranjeras, tanto de carácter oficial como formal, sin embargo, con antelación se logró recabar una diversidad de material impreso, que junto al material digital fue sometido a una detallada revisión y lectura que resultó en una selección acorde a los objetivos planteados y al criterio de búsqueda del presente trabajo investigativo. Una vez procesada y discutida toda la información se procedió a presentar esta disertación que inicia con la descripción de cómo se ha manifestado recientemente en Ecuador la internacionalización de sus Universidades.

Tal y como ya se puede deducir, la naturaleza del presente trabajo es documental, de tipo descriptivo y a nivel exploratorio, puesto que la fuente primordial de la información obtenida es mayormente a través de documentos escritos, con los que se persigue básicamente aportar una descripción de una situación particular acontecida en el contexto sociocultural y educativo de Ecuador, teniendo claro desde un principio que, independientemente del cúmulo de información leído y analizado, sólo se pretende realizar un acercamiento a un tema que sirva de información general al lector.

De acuerdo a lo afirmado por (Ríos, 2017) respecto a la Tipología de las Investigaciones, una investigación es documental cuando se “....recurre a la consulta de documentos para obtener sus resultados.". En cuanto a la profundidad que se pretende alcanzar, el mismo autor refiere que 


\section{La Universidad Ecuatoriana ante las nuevas Profesiones}

Vol. 2, núm. 2., (2018)

Sofía Lissette Pacherres Nolivos; Eugenia Estefanía Alvarado Palma; Irma Elizabeth Naranjo Peña; David Benavides López

se está en presencia de una investigación de tipo descriptiva cuando se “...busca encontrar las características, comportamiento y propiedades del objeto de estudio, ya sea en el presente o en el futuro..."(p.81). Ahora, según Rojas (2002), se habla de estudios exploratorios cuando la investigación "Se refiere a un primer acercamiento que el investigador tiene con el problema o fenómeno que estudia, con el objeto de realizar un análisis descriptivo del mismo...". El mismo autor asegura que "El contacto con el objeto de estudio puede hacerse por diferentes vías: observación directa y observación indirecta (por ejemplo, entrevista a informantes clave), y por medio del análisis preliminar de documentos diversos que traten sobre el tema” (p.144). Todo ello fundamenta lo antes afirmado respecto a la metodología aplicada en el presente trabajo investigativo.

Es importante destacar que el acceso a la información digital se efectuó a través del motor de búsqueda de Google Académico ${ }^{\circledR}$ y Google $^{\circledR}$. La búsqueda de dicha información se hizo de manera libre y aleatoria, teniendo como criterio la nuevas profeciones ofrecidas en las universidades de Ecuador, llegando a seleccionar del total del material disponible y consultado, sólo el contenido, que desde nuestro punto de vista, resultó ser más adecuado y relevante.

\section{Resultados.}

\section{Ámbito}

Es necesario iniciar esta parte compartiendo la idea de Macías \& Mendoza (2016) puesto que en su obra se hace referencia a "La Era de la Complejidad", que no es más que una forma de llamar al conjunto de factores que posibilitan la visualización de una nueva forma de sociedad demandante, lo que consecuentemente se traduce en, educar de manera innovadora y modelación 


\section{La Universidad Ecuatoriana ante las nuevas Profesiones}

Vol. 2, núm. 2., (2018)

Sofía Lissette Pacherres Nolivos; Eugenia Estefanía Alvarado Palma; Irma Elizabeth Naranjo Peña; David Benavides López

de las personas que forman parte de esta sociedad moderna. En esa misma exposición, se deja claro que a la par de los cambios de un sistema educativo debe producirse también el de la sociedad que constituye dicho sistema, y que por el hecho de que esos cambios sean complejos, no se está garantizado que sean completamente determinantes, ni mucho menos perennes. Seguidamente, los autores continúan y complementan esa idea haciendo referencia a Guillaumín, (2001) quien señala que:

La Universidad que se quiere instaurar y que parece reunir diversos rasgos, tales como: uso intensivo de tecnologías informáticas; la incorporación de la virtualidad en la enseñanza; mayor apertura al entorno, sobre todo a los sectores productivos modernos y al ámbito internacional; carreras cortas y curriculas flexibles para ajustarse a los mercados emergentes; énfasis en el desarrollo tecnológico; venta de servicios como nueva vía de financiamiento; adopción de conceptos de excelencia y competitividad del mundo empresarial. (p. 2-3)

Lo que se quiere dar a entender con este concepto, según lo que se entiende de (Macías \& Mendoza, 2016), es precisamente la complejidad a la que debe responder la Universidad como institución transformadora por excelencia de una sociedad, para proporcionar a través de su estructura académica la respuesta a la demanda del contexto en que se encuentra.

A través esta investigación, se ha llegado a comprender que en America Latina esa es la pretensión, y Ecuador no es la excepción, puesto que los esfuerzos que últimamente se están adelantando a través de la gestión del Estado en esa materia, están orientados a solventar esa tendencia, claro, eso es hasta cierto punto, ya que lo logrado hasta ahora no es suficiente, ni lo 


\section{La Universidad Ecuatoriana ante las nuevas Profesiones}

Vol. 2, núm. 2., (2018)

Sofía Lissette Pacherres Nolivos; Eugenia Estefanía Alvarado Palma; Irma Elizabeth Naranjo Peña; David Benavides López

será a lo largo del tiempo, por una parte debido al constante impacto que generan los avances tecnológicos a nivel mundial, y por otro, a los cambios que se producen en el propios contexto social, económico, geográfico y cultural, razones por las cuales siempre será necesario la constante revisión, mejoramiento y adaptación de su sistema educativo en general para alcanzar el desarrollo sostenido que toda sociedad y país aspira.

\section{Respecto al marco legal ecuatoriano}

Una de las puntas de lanza que demuestra la intención del gobierno ecuatoriano en acertar al mejoramiento de su sistema educativo superior, enfoque del presente trabajo, podría decirse que inició a partir de la reforma a su Carta Magna en el año 2008, cuando en el artículo 350 se estableció que:

El sistema de educación superior tiene como finalidad la formación académica y profesional con visión científica y humanista; la investigación científica y tecnológica; la innovación, promoción, desarrollo y difusión de los saberes y las culturas; la construcción de soluciones para los problemas del país, en relación con los objetivos del régimen de desarrollo. (Const., 2008, Art. 350)

También es importante mencionar que, en lo que compete al área educativa, en el mismo instrumento jurídico se deja claro, entre otras cosas, que:

- La educación es un derecho de las personas a lo largo de su vida y un deber ineludible e inexcusable del Estado. Constituye un área prioritaria de la política pública y de la inversión estatal, garantía de la igualdad e inclusión social y condición indispensable 


\section{La Universidad Ecuatoriana ante las nuevas Profesiones}

Vol. 2, núm. 2., (2018)

Sofía Lissette Pacherres Nolivos; Eugenia Estefanía Alvarado Palma; Irma Elizabeth Naranjo Peña; David Benavides López

para el buen vivir. (Const., 2008, Art.26)

- La educación se centrará en el desarrollo de competencias y capacidades para crear y trabajar. (Const., 2008, Art.27)

- La educación responderá al interés público, y no estará al servicio de intereses individuales y corporativos. (Const., 2008, Art.28)

- El Estado garantizará la libertad de enseñanza, la libertad de cátedra en la educación superior. (Const., 2008, Art.29)

- Que el Sistema de Educación Superior estará articulado al sistema nacional de educación y al Plan Nacional de Desarrollo y que dicho sistema se regirá por los principios de autonomía responsable, cogobierno, igualdad de oportunidades, calidad, pertinencia, integralidad, autodeterminación para la producción del pensamiento y conocimiento, en el marco del diálogo de saberes, pensamiento universal y producción científica tecnológica global (Const., 2008, Art.351)

En concordancia con ese marco jurídico, en 2010 se sanciona la Ley Orgánica de Educación Superior, documento que le dio operatividad al mencionado marco y con el que, en relación al tema aquí planteado, se fortalece y resalta el rol del Estado, y además se establecen según Menéndez \& Cevallos (2017), tanto las bases jurídicas para "la conformación de nuevos órganos de planificación, regulación y coordinación de la Educación Superior”, como “una estructura de educación superior que contribuya a la transformación de la sociedad, a su estructura social, productiva y ambiental, formando profesionales y académicos con capacidades 


\section{La Universidad Ecuatoriana ante las nuevas Profesiones}

Vol. 2, núm. 2., (2018)

Sofía Lissette Pacherres Nolivos; Eugenia Estefanía Alvarado Palma; Irma Elizabeth Naranjo Peña; David Benavides López

y conocimientos que respondan a las necesidades de desarrollo" (p. 68)

Esos aspectos se vinculan, en términos generales, con la idea de la internacionalización de la educación superior, y consecuentemente, con los retos y desafíos de la nueva gestión universitaria en contextos de complejidad e incertidumbre que, según (Cevallos, 2016) son: Democracia Cognitiva, por la adquisición de conocimientos para satisfacer necesidades, garantizar derechos y potenciar capacidades; Innovación Social, vinculado a la creación de las plataformas interinstitucionales, a la creación de modelos de gestión sistémica y a la planificación por dominios científicos, tecnológicos y humanísticos que se articulen a las funciones sustantivas; Pertinencia, en pro del desarrollo de capacidades para la Economía Social del Conocimiento y de lograr aprendizajes significativos y relevantes centrados en la experiencia de indagación, reflexividad y producción crítica y creativa de saberes para la resolución de problemas; la Calidad Educativa, que se pretende garantizar como bien público y eje de la transformación de la sociedad, a través de modelos estratégicos y prospectivos; y finalmente, la Consolidación del Sistema de Educación Superior, pretendido por medio del desarrollo del proyecto nacional y la configuración de redes del conocimiento y académicas.

Para cerrar lo que hasta aquí se ha explicado, se consideró pertinente destacar el hecho de que:

El reto de la universidad pública y privada, así como el de los estudiantes, está en ofrecer y adquirir competencias y destrezas profesionales de acuerdo a los requerimientos del mercado laboral, que demanda profesionales que sepan que es una minería de datos, cómo funcionan los sistemas de simulación, qué es la inteligencia artificial y que tengan 


\section{La Universidad Ecuatoriana ante las nuevas Profesiones}

Vol. 2, núm. 2., (2018)

Sofía Lissette Pacherres Nolivos; Eugenia Estefanía Alvarado Palma; Irma Elizabeth Naranjo Peña; David Benavides López

la suficiente capacidad de seleccionar información de relevancia con rapidez y calidad para tomar. (ekosnegocios.com, 2015, p.50)

Las universidades públicas y privadas se están adecuado sobre la marcha para, por un lado, cumplir con todas las exigencias y reformas públicas, así como invertir en infraestructura, contratación de talento y servicios, para brindar un servicio integral que apunte hacia la calidad total. Todos los esfuerzos están volcados en forjar universidades adecuadas y aptas para brindar un servicio integral y educación de calidad. (ekosnegocios.com, 2015, p.51)

\section{Nuevas Profesiones}

García (2012) dice que “el impacto de la internacionalización tiene implicaciones sociales que conllevan a la unificación de mercados, sociedades y culturas”. Así mismo asegura que en el marco de la globalización (fenómeno del cual Ecuador no es ajeno), "las profesiones se han visto influenciadas enérgicamente, y en razón de ello nacen nuevas profesiones y la reorientación de otras ya existentes".

Esta misma autora hace un interesante aporte en su obra al mencionar y describir las competencias/habilidades que a futuro demandaría el mercado laboral, teniendo ella como base la interpretación propia de un estudio denominado "Future Work Skills 2020", que fue ejecutado por Institute for the Future (IFTF) para The University of Phoenix Research Institute. En dicho estudio determinan la existencia de factores de cambios tales como: la longevidad extrema, porque el aumento de vida en las personas cambia la naturaleza de las carreras, de los conocimientos y del saber, por tanto, las organizaciones tendrán que volver a reflexionar la 


\section{La Universidad Ecuatoriana ante las nuevas Profesiones}

Vol. 2, núm. 2., (2018)

Sofía Lissette Pacherres Nolivos; Eugenia Estefanía Alvarado Palma; Irma Elizabeth Naranjo Peña; David Benavides López

trayectoria de las carreras tradicionales creando más diversidad y flexibilidad; la existencia de las máquinas y sistemas inteligentes, ya que la automatización trastocan y cambian el rumbo repetitivo de las tareas que realizan los trabajadores; mundo computacional, debido al gran incremento de sensores hacen del mundo un sistema programable; nиеvo medio de comunicación, las nuevas tecnologías multimedia están provocando una transformación en la comunicación y se está participando en el desarrollando un nuevo lenguaje para la misma; organizaciones superestructuradas, las nuevas tecnologías están conduciéndonos a una reorganización sin precedentes, de cómo producimos y creamos valor; y por ultimo; mundo globalmente conectado, la globalización es, a largo plazo, fuerte tendencia hacia el "intercambio" e "integración" geográfica. Entonces, es a partir de éstos factores que la autora comenta sobre las competencias y habilidades que el mercado laboral demanda a futuro, entre ellas están:

- Dar sentido: Habilidad para determinar el significado más profundo de lo que está siendo expresado.

- Inteligencia social: Habilidad para conectarse con otros de forma directa.

- Pensamiento original y adaptativo: Es la habilidad para pensar y descubrir soluciones y respuestas más allá de lo que la memoria dicta o basándose en reglas.

- Competencia transcultural: Es la capacidad para intervenir en diferentes escenarios culturales.

- Pensamiento computacional: Es la habilidad para entender datos abstractos y comprender los datos basados en el razonamiento. 


\section{La Universidad Ecuatoriana ante las nuevas Profesiones}

Vol. 2, núm. 2., (2018)

Sofía Lissette Pacherres Nolivos; Eugenia Estefanía Alvarado Palma; Irma Elizabeth Naranjo Peña; David Benavides López

- Formación sobre nuevos "Media": Es la capacidad para evaluar críticamente y desarrollar contenido en las nuevas formas de "los Media"(Internet), y aprovecharlos para la comunicación persuasiva.

- Transdisciplinariedad: Formación y habilidad para comprender conceptos a través de múltiples disciplinas.

- Mentalidad para el diseño: Habilidad para desarrollar tareas y procesos de trabajo para obtener resultados deseados.

- Gestión cognitiva: Habilidad para discriminar y filtrar información por su importancia y veracidad.

- Colaboración virtual: Habilidad para trabajar productivamente, manteniendo el compromiso, y demostrando la presencia como miembro que forma parte de un equipo virtual.

En este mismo orden de ideas puede hacerse mención entonces a las tendencias profesionales que están surgiendo mayormente por el surgimiento y rápido crecimiento de las nuevas tecnologías, más que todo vinculadas al entorno digital.

De acuerdo a la noticia publicada en el portal de Universia Colombia (2018):

El mercado laboral demanda empresas digitalizadas y profesionales capaces de comprender el entorno digital y adaptarse a este, por lo tanto, los centros educativos tienen una nueva misión por delante: adaptar sus conocimientos a las necesidades de este 


\section{La Universidad Ecuatoriana ante las nuevas Profesiones}

Vol. 2, núm. 2., (2018)

Sofía Lissette Pacherres Nolivos; Eugenia Estefanía Alvarado Palma; Irma Elizabeth Naranjo Peña; David Benavides López

mercado tan innovador como cambiante.

Así como las ideas deben pensarse en digital y las formas de trabajo deben adecuarse a las nuevas modalidades que permite la tecnología, es necesario que las carreras y estudios universitarios se adapten a esta nueva realidad.

En esa misma entrega se deja claro que dentro del surgimiento de las nuevas profesiones, resaltan las denominadas Profesiones Digitales, siendo éstas las que precisamente sacan el máximo provecho de las nuevas tecnologías. Así mismo, afirman que estas profesiones no necesaria mente son enseñadas en universidades debido a que son primordialmente carreras técnicas, de programas breves y adaptados a las últimas tendencias tecnológicas y educativas.

A manera de ejemplo, a continuación se mencionan las profesiones que en el referido artículo dicen que constituyen las nuevas profesiones, y se complementa dicha lista enunciando otras carreras mencionadas igualmente en un artículo informativo de la misma fuente, con un tema muy similar, pero publicados en los años 2016:

- Experto en normativas digitales.

- Científico de Datos.

- Programador.

- Analistas y programadores del Internet de las Cosas.

- Arquitectos de realidad virtual. 


\section{La Universidad Ecuatoriana ante las nuevas Profesiones}

Vol. 2, núm. 2., (2018)

Sofía Lissette Pacherres Nolivos; Eugenia Estefanía Alvarado Palma; Irma Elizabeth Naranjo Peña; David Benavides López

- Diseñador de órganos.

- Robotista.

- Diseñador de redes neuronales artificiales.

- Terapeuta de empatía artificial

- Impresor 3D.

- Protésico robótico

- Ingenieros de nanorobots médicos. (Ésta evidentemente no sería técnica)

- Abogado especializado en drones y ciberseguridad.

Para concluir con esta sección y sin detrimento de los tantos logros alcanzados, es imperioso mencionar que, tras la ardua tarea de búsqueda, selección, lectura e interpretación de documentación pertinente en el tema, se encontró sólo un verdadero ejemplo que relaciona a la universidad ecuatoriana con una nueva profesión, y es el mencionado por Giudice \& Haquehua (2014), quienes informaron a manera de ejemplo, sobre una nuevas carreras ofrecida en Ecuador denominada "Ingeniería del Litoral", estudios ofrecidos a través de la Universidad del Litoral (Privada), destacando que la misma, a parte de haberse convertido en todo un éxito, era cofinanciada por la Organización de las Naciones Unidas (ONU). Finalizan agregando que los graduados en esa carrera se encargaban de asegurar la vida de la fauna y flora marina del Golfo de Guayaquil, e igualmente son aptos para llevar adelante la construcción de defensas (Muelles) contra los tsunamis y arenar las playas del Ecuador. 


\section{La Universidad Ecuatoriana ante las nuevas Profesiones}

Vol. 2, núm. 2., (2018)

Sofía Lissette Pacherres Nolivos; Eugenia Estefanía Alvarado Palma; Irma Elizabeth Naranjo Peña; David Benavides López

\section{Conclusiones.}

Luego de discernir sobre toda la literatura consultada y explicada, se hace indispensable iniciar estas conclusiones destacando que, independientemente de que en el área de educación superior Ecuador hayan logrado metas comparables a las de destacadas referentes extranjeros, por ejemplo, muy similares a las de los miembros de la Organización para la Cooperación y el Desarrollo Económico (OCDE) al término del 2015 (tales como: extensión de la escolarización, universalización de la enseñanza, formación del profesorado, organización de los centros educativos, metodologías utilizadas, profesionalización de la gestión, infraestructuras disponibles, recursos dedicados, entre otros) no deja de ser cierto lo expuesto por Cobos (2018) quien afirmó que, aparte de tantos, uno de los retos fundamentales a superar a nivel educativo en Ecuador, está basado en alcanzar un mayor nivel investigativo en las universidades, así como también es necesario un mayor proceso de vinculación internacional, puesto que, a pesar de los avances y los esfuerzos, todavía hace falta que (las universidades) estén aún más relacionadas con el sistema educativo global. (p. 30)

Al término de la presente investigación, lo que se puedo evidenciar respecto a la política que el Estado ecuatoriano adopta frente al surgimiento de las nuevas profesiones es que ese fenómeno lo tratan (al parecer) sólo desde el marco del "Proyecto de Reconversión de la Formación Técnica y Tecnológica Superior Pública”, el cual contribuye en el cumplimiento del objetivo 2 del Plan Nacional para el Buen Vivir, referente al desarrollo de capacidades y potencialidades ciudadanas; y está relacionado específicamente con la política 2.5 que determina la necesidad de fortalecer la educación superior con visión científica y humanista, articulada a los objetivos para el Buen Vivir. En otras palabras, con dicho proyecto se persigue: Fortalecer el 


\section{La Universidad Ecuatoriana ante las nuevas Profesiones}

Vol. 2, núm. 2., (2018)

Sofía Lissette Pacherres Nolivos; Eugenia Estefanía Alvarado Palma; Irma Elizabeth Naranjo Peña; David Benavides López

sistema de educación superior no universitaria, transformando física y académicamente a los institutos superiores técnicos y tecnológicos públicos (ISTTP), alineado a las necesidades del país, y al Plan Nacional para el Buen Vivir; Reconvertir la formación técnica y tecnológica superior del país respecto a las carreras ligadas a los sectores estratégicos, prioritarios y de servicios públicos esenciales, con el fin de aportar al cambio de la matriz productiva del Ecuador.

Finalmente se considera importante hacer eco de la premisa enunciada por Cevallos, presidente de la Comisión Permanente de Universidades y Escuelas Politécnicas del Consejo de Educación Superior de Ecuador, al exponer sobre la Internacionalización de la Educación Superior en Ecuador, en Huesca, España, no sólo por la pertinencia, sino que a pesar de que fue hace poco más de un año, no pierde vigencia: “Transformar la Universidad para transformar la sociedad y el país” (2016)

\section{Referencias Bibliográficas}

Cevallos, M. (09 de 2016). http://www.auip.org/es/. Obtenido de http://www.auip.org/es/: https://www.auip.org/images/stories/DATOS/PDF/seminarios/unia/Ponencia_Marcelo_C evallos_Jornadas_UNIA.pdf

Cobos, J. (2018). La Educación Superior en el Ecuador. Situación actual y perspectivas de futuro desde el contexto de las Tecnologías de la Información y la Comunicación. International Studies on Law and Education (29/30), 29 - 40. Obtenido de http://www.hottopos.com/isle29_30/29-40Cobos.pdf

Const. (20 de 10 de 2008). Constitucion de la Republica del Ecuador / Titulo VII: Del Régimen del Buen Vivir. Sección Primera: Educación. Ecuador. Obtenido de https://www.oas.org/juridico/pdfs/mesicic4_ecu_const.pdf

EcuadorUniversitario.com. (27 de 06 de 2016). http://ecuadoruniversitario.com. Obtenido de http://ecuadoruniversitario.com: http://ecuadoruniversitario.com/opinion/la-educacionsuperior-en-el-ecuador/ 


\section{La Universidad Ecuatoriana ante las nuevas Profesiones}

Vol. 2, núm. 2., (2018)

Sofía Lissette Pacherres Nolivos; Eugenia Estefanía Alvarado Palma; Irma Elizabeth Naranjo Peña; David Benavides López

ekosnegocios.com. (05 de 2015). Especial de Educación Superior en Ecuador. Ekos(253), 124. Obtenido de http://www.ekosnegocios.com/revista/pdf/253.pdf

García, T. (16 de 04 de 2012). educaweb.com. Obtenido de educaweb.com: https://www.educaweb.com/noticia/2012/04/16/nuevas-tecnologias-crean-nuevasprofesiones-5439/

Giudice, V., \& Haquehua, O. (2014). Nuevas profesiones universitarias Siglo XXI. Pensamiento $\begin{array}{llllll}\text { Crítico, } & 19(2), & 139 & - & 147 . & O b t e n i d o \\ \text { de }\end{array}$ http://revistasinvestigacion.unmsm.edu.pe/index.php/econo/article/viewFile/11108/9985

Macías, M., \& Mendoza, F. (2016). Desafíos del sistema de educación superior en Ecuador para la era de la complejidad. Foro Educacional(26), 89 - 114. Obtenido de https://dialnet.unirioja.es/servlet/articulo?codigo $=6429507$

Menéndez, E., \& Cevallos, D. (12 de 2017). EL VÍNCULO DE LA EDUCACIÓN SUPERIOR CON EL CAMBIO DE LA MATRIZ PRODUCTIVA EN EL ECUADOR. Ciencias Pedagógicas e Innovacion, V(3), 98 - 74. doi:http://dx.doi.org/10.26423/rcpi.v5i3.195

Ríos, R. (09 de 2017). www.eumed.net. (E. Grupo de investigación (SEJ 309) eumed.net de la Universidad de Málaga, Ed.) Obtenido de www.eumed.net: http://www.eumed.net/libros/libro.php?id=1662

Rojas, R. (2002). Investigación Social. Teoría y Praxis. Mexico, D.F., Maxico: Plaza y Valdéz, S.A. de C.V. 